\title{
PRÁTICA DE ATIVIDADE FÍSICA: CORRELAÇÃO ENTRE A PRÁTICA NA INFÂNCIA E NA VIDA ADULTA
}

Nélio de Paula, Lorrany Oliveira Scardazzi Silva, Welligton Roberto Hogera Rodrigues, Marcelo Jose Alves, Mateus de Carvalho, Diego Giulliano Destro Christofaro

Curso de Educação Física da UNOESTE, Presidente Prudente, SP.

\section{RESUMO}

O objetivo deste estudo foi verificar a prevalência da prática de atividade física na vida adulta resultante de experiência na fase da infância e adolescência e, o que isso pode representar na saúde da vida adulta, principalmente na pressão arterial. Os dados foram coletados por meio de questionário fechado e aferição da pressão arterial e, assim comparado a inter-relação entre os dados e a co-relação de que a prática de atividade física nas fases da infância e adolescência pode resultar na alteração da pressão arterial na vida adulta.

Palavras-chave: Infância, adolescência, pressão arterial, atividade física.

\section{PRACTICE OF PHYSICAL ACTIVITY: CORRELATION BETWEEN THE PRACTICE IN INFANCY AND THE ADULT LIFE}

\section{ABSTRACT}

The aim of this study was to verify the prevalence of the physical activity practice in the adult life as a result of experience in the childhood and adolescence phase and, what it can represent in the health of the life adult, mainly in the arterial pressure. The data were collected through closed questionnaire and gauging of arterial pressure and, thus compared the inter-relation between the data and the co-relation of what the practice of physical activity in the childhood and adolescence phases can result in the alteration of the arterial pressure in the adult life.

Keywords: Childhood, adolescence, arterial pressure, physical activity. 


\section{INTRODUÇÃO}

Até o inicio do século $X X$ as doenças que mais causavam óbitos eram aquelas com características infecto-contagiosas, tais como: malária, sarampo, tuberculose, dentre outras (BUCHALA \& WALDMAN, 2003). Porém, com as melhorias de saneamento básico e o avanço da área médica, a prevalência desses tipos de enfermidades diminuiu consideravelmente. Outro fator que contribuiu para a redução na ocorrência deste tipo de doença foram os avanços tecnológicos.

Além de contribuir para a redução da mortalidade, o avanço tecnológico propiciou mais comodidade e conforto para as pessoas, facilitando dessa forma atividades cotidianas que requeriam certo nível de esforço. Contudo esses avanços não foram apenas benéficos, pois, a comodidade e facilidades proporcionadas por esse fator contribuíram para a diminuição da atividade física diária e, conseqüentemente, para a instalação de doenças hipocinéticas. Entre as doenças que podem ser consideradas fatores de risco destacam-se o diabetes mellitus (SCHAAN, 2004), a hipertensão arterial (GUS et al., 2004), dislipidemias (SILVA et al., 2006) e o excesso de gordura corporal (SPOSITO et al., 2007).

A falta da prática de atividades físicas tem contribuído para o aumento desse problema, uma vez que o sedentarismo tem sido atrelado aos fatores de risco cardiovasculares, bem como, os eventos cardíacos em si.

Em estudo recente, Fernandes e Zanesco (2010) identificaram que:

\begin{abstract}
"64,5\% da população adulta de oito diferentes cidades do estado de São Paulo foram considerados inativos. Contudo o mesmo estudo apontou que a prática de atividade física realizada na infância e adolescência parece contribuir para a prevenção de doenças cardiovasculares durante a vida adulta."
\end{abstract}

Dessa forma o objetivo do presente estudo foi estimar a prevalência hipertensão arterial em universitários do curso de Educação Física de uma universidade do interior do Estado de São Paulo e verificar a relação entre a prática de atividade física na infância com os valores de pressão arterial nessa população.

\section{MÉTODOS}

\section{Amostra}

A amostra foi selecionada por conveniência e foi composta por 100 adultos maiores de 18 anos estudantes do curso de Educação Física de uma universidade do interior do Estado de São Paulo. Participaram do estudo 64 homens e 36 mulheres com idade entre 19 a 45 anos. Todos os sujeitos que aceitaram a participar do estudo assinaram um termo de consentimento livre esclarecido. $O$ presente estudo foi avaliado e aprovado pelo Comitê de Ética e Pesquisa da Universidade do Oeste Paulista (UNOESTE).

\section{Pressão Arterial}

As medidas de pressão arterial foram mensuradas em uma sala reservada. Para tanto, os indivíduos permaneceram sentados em repouso por cinco minutos, com o dorso encostado na cadeira e com braço e pernas relaxadas seguindo as recomendações da VI Diretrizes Brasileiras de Hipertensão Arterial (2010), para então ser realizada a primeira aferição. A segunda aferição da medida da pressão arterial ocorreu após dois minutos da realização da primeira.

Para a avaliação da medida da pressão arterial foi utilizado um aparelho oscilométrico da marca Omron modelo HEM 742. As medidas foram realizadas no braço direito do indivíduo. Os sujeitos com pressão arterial sistólica superior a $140 \mathrm{mmHg}$ ou pressão arterial diastólica acima de $90 \mathrm{mmHg}$ foram considerados com hipertensão arterial de acordo com as recomendações da VI Diretrizes Brasileiras de Hipertensão (2010). 


\section{Prática continuada de atividade física}

A prática de atividade continuada de atividade física na infância foi verificada mediante as seguintes perguntas: "Fora da escola, na infância (7-10 anos) você praticou alguma atividade esportiva (supervisionada) por um ano"?

\section{ANÁLISE ESTATÍSTICA}

$\mathrm{Na}$ análise estatística dos dados foi realizado o teste paramétrico de Shapiro Wilks. No caso dos dados que se enquadraram no modelo Gaussiano de distribuição, a análise descritiva foi expressa em média e desvio-padrão. A associação entre as variáveis dependentes e independentes utilizou o teste do Qui-quadrado com correção de Yates.

\section{RESULTADOS}

$\mathrm{Na}$ Tabela 1 são apresentadas as características da amostra. Os sujeitos do sexo masculino não diferiram em relação à idade dos sujeitos do sexo feminino $(p=0,812)$. Não houve diferença também quando se considerou a pressão arterial sistólica $(p=0,571)$ ou a pressão arterial diastólica $(p=0,152)$.
Tabela 1. Característica da amostra e valores da pressão arterial sistólica (PAS) e pressão arterial diastólica (PAD) (média $\pm \mathrm{DP})$.

\begin{tabular}{lcc}
\hline & $\begin{array}{c}\text { Masculino } \\
(n=64)\end{array}$ & $\begin{array}{c}\text { Feminino } \\
(n=36)\end{array}$ \\
\hline Idade & $24,34 \pm 4,87$ & $24,61 \pm 5,64$ \\
PAS & $120,97 \pm 18,00$ & $128,75 \pm 21,74$ \\
PAD & $68,51 \pm 8,39$ & $11,26 \pm 71,64$ \\
\hline
\end{tabular}

Na Tabela 2 é apresentada a informação da relação da prática de atividade física na infância e adolescência. Após ter sido realizada a análise estatística não foi verificada relação significante entre os sujeitos que relataram fazer atividade física na infância e os valores de pressão arterial na vida adulta.

Tabela 2. Relação entre a prática de atividade física na infância e os valores de pressão arterial na vida adulta.

\begin{tabular}{lcccc}
\hline \multicolumn{5}{c}{ Pressão arterial } \\
\hline & $\begin{array}{c}\text { Normal } \\
(\%)\end{array}$ & $\begin{array}{c}\text { Hipertenso } \\
(\%)\end{array}$ & $\begin{array}{c}\text { Total } \\
(\%)\end{array}$ & $\mathbf{P}$ \\
Não & 81,8 & 18,2 & 100 & \\
Praticava & & & & 1,00 \\
Praticava & 79,5 & 20,5 & 100 & \\
\hline
\end{tabular}

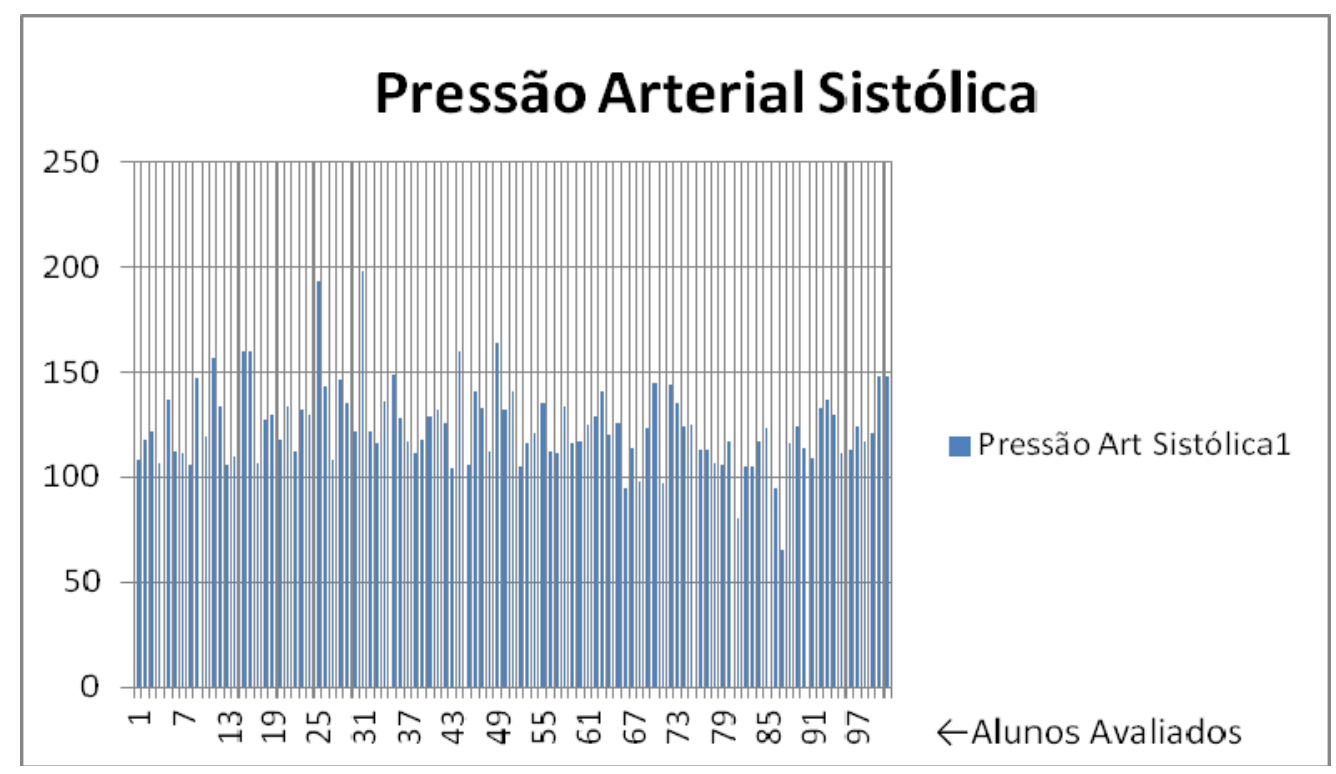

Figura 1. Variação da Pressão Arterial Sistólica nos 100 sujeitos analizados durante o estudo, cuja média foi de 120,97 mmHg. Com base na verificação padrão de 120 por 80 mmHg, o desvio padão foi de 18,00 (dp). 


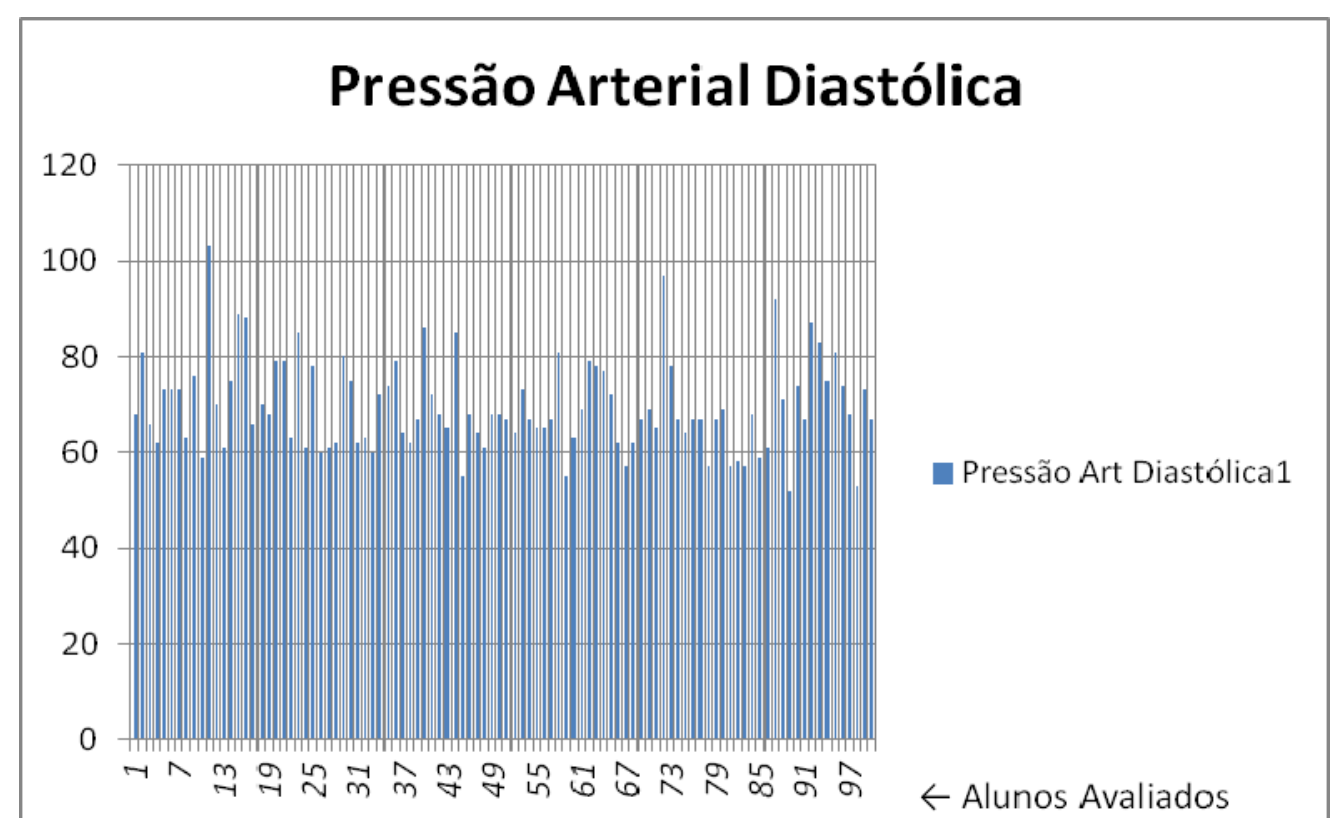

Figura 2. Variação da Pressão Arterial Diastólica nos 100 sujeitos analizados durante o estudo, cuja média foi de 68,51 mmHg. Com base na verificação padrão de 120 por 80 mmHg, o desvio padão foi de 8,39 (dp).

\section{DISCUSSÃO}

Estudo descritivo de característica analítica que teve como objetivo verificar uma possível relação entre a prática de atividade física na infância e valores de pressão arterial na vida adulta. Dentre os 100 alunos avaliados, 64 eram homens e 36 eram mulheres, os quais têm na sua vida diária atividades de nível funcional ou praticam algum tipo de atividade física de leve a moderado esforço físico. Porém, no presente estudo apenas a prática de atividade física na infância foi considerada.

Conforme pesquisa realizada pela $\mathrm{VI}$ Diretrizes Brasileiras de Hipertensão (2006) foram considerados, com pressão alta, sujeitos com pressão arterial sistólica acima de $140 \mathrm{mmHg}$ e/ou com pressão arterial diastólica acima de 80 $\mathrm{mmHg}$. A variável pressão arterial tem sido amplamente estudada pelo fato de que vários estudos têm reportado elevada prevalência de hipertensão arterial no mundo.

Além desta, percebemos através de estudos realizados por Costa et al. (2007) com 1968 pessoas na região sul brasileira, onde foi detectada uma prevalência de $23,6 \%$ de brasileiros hipertensos.

E em recente estudo de base populacional realizado em diversas regiões dos Estados Unidos, Keenan e Rosendorf (2011) relataram que aproximadamente $30 \%$ dos americanos foram considerados hipertensos.

No que se refere à prática de atividade física, alguns estudos, como de Kokkinos (2011), tem relatado elevada prevalência de sedentarismo na população brasileira, fator esse preocupante, uma vez que a prática insuficiente de atividade física é associada a fatores de risco cardiovasculares.

Hallal et al. (2003), após investigarem mais de 3.000 pessoas no município de Pelotas, no estado do Rio Grande do Sul, observaram que aproximadamente $40 \%$ dos sujeitos avaliados foram considerados sedentários.

Em um estudo realizado por Fernandes e Zanesco (2010) em quatro cidades do Estado de São Paulo (Presidente Prudente. Rio Claro, Bauru e São Paulo) foi observado que 58,4\% dos homens e $68,3 \%$ das mulheres foram considerados sedentários após a análise da 
prática habitual de atividade física mediante 0 questionário de Baecke et al. (1982).

No presente estudo presumimos a importância da variável atividade física com relação à pressão arterial. Pois segundo Fernandes e Zanesco (2010) a prática de atividade física realizada na infância e adolescência parece contribuir para a prevenção de doenças cardiovasculares durante a vida adulta. Com relação aos resultados do nosso estudo, podemos observar que mesmo entre os alunos que praticaram atividade física na infância, que representaram $79,5 \%$ da amostra, tivemos um numero acentuado de pessoas com a pressão arterial elevada, aproximando-se dos valores reportados por Costa et al. (2007) e por Kenan e Rosendorf (2011). Porém, em nosso estudo a prevalência de pressão arterial elevada não diferiu entre aqueles sujeitos que relataram fazer atividade física na infância quando comparados aos sujeitos que não relataram fazer atividade física na infância, 20,5\% versus 18,5\%, respectivamente.

A relevância no resultado foi pouco significativa devido, principalmente, a pouca diferença entre as idades dos adultos que foram submetidos ao teste e também tendo em vista que se utilizou somente os alunos do curso da Educação Física, pois a comparação teria um melhor parâmetro se utilizássemos outros cursos, não só com alunos mais velhos como com um índice maior de inatividade física, diferença de nível sócio econômico e cultural, fumantes ou sedentários obesos. Além disso, destaca-se a proximidade pelo gosto da prática esportivos geralmente característicos dos alunos do curso de Educação Física, o que contribuiu para a homogeneidade detectada na presente amostra.

\section{CONCLUSÃO}

Este estudo servirá como base para outras verificações dentro do mesmo processo podendo ainda utilizar, outros métodos de medição como a antropometria, o IMC, teste de $\mathrm{VO}_{2}$ máx., entre outros destinados a verificação de sedentarismo e obesidade da vida da criança e/ou do adolescente até a vida adulta. Conclui-se que o presente estudo contribui para uma análise de nível moderada dentro do assunto, mas que, com uma valência relevante dentro da área estudada, já que poderia ter sido abrangido um grupo bem maior de pessoas e diversidades de observações antropométricas e fisiológicas no estudo, relacionadas à prática de atividade física na infância.

\section{CONFLITO DE INTERESSE}

Os autores declaram não haver qualquer potencial conflito de interesse que possa interferir na imparcialidade deste trabalho científico.

\section{REFERÊNCIAS}

Baecke JA, Burema J, Frijters JE. A short questionnaire for the measurement of habitual physical activity in epidemiological studies. Am J Clin Nutr. 1982;36:936-42.

Buchala CM, Waldman EA. A mortalidade por doenças infecciosas no início e no final do século XX no Município de São Paulo. Rev Bras Epidemiol. 2003;6:335-344. DOI http://dx.doi.org/10.1590/S1415790X2003000400008

Costa JS, Barcellos FC, Sclowitz ML, Sclowitz IK, Castanheira M, Olinto MT. Hypertension prevalence and its associated risk factors in adults: a population-based study in Pelotas. Arq Bras Cardiol. 2007;88(1):59-65. DOI http://dx.doi.org/10.1590/S0066782X2007000100010

Fernandes RA, Zanesco A. Early physical activity promotes lower prevalence of chronic diseases in adulthood. Hypertens Res. 2010;33(9):926-31. DOI http://dx.doi.org/10.1038/hr.2010.106

Gus I, Harzheim E, Zaslavsky C, Medina C, Gus M. Prevalência, reconhecimento e controle da hipertensão arterial sistêmica no estado do Rio Grande do Sul. Arq Bras Cardiol. 2004;83 (5):4248. DOI http://dx.doi.org/10.1590/S0066782X2004001700009 
Hallal PC, Victora CG, Wells JC, Lima RC. Physical inactivity: prevalence and associated variables in Brazilian adults. Med Sci Sports Exerc. 2003;35:1894-900. DOI http://dx.doi.org/10.1249/01.MSS.0000093615.33 $\underline{774.0 \mathrm{E}}$

Keenan NL, Rosendorf KA. National Center for Chronic Disease Prevention and Health Promotion, CDC. Prevalence of Hypertension and Controlled Hypertension - United States, 20052008. MMWR Surveill Summ. 2011;60(SU-1):9497.

Kokkinos $P$, Sheriff $H$, Kheirbek R. Physical inactivity and mortality risk. Cardiol Res Practice. 2011;924-945.

Schaan B, Harzheim E, Gus I. Perfil de risco cardíaco no diabetes mellitus e na glicemia de jejum alterada. Rev Saúde Pub. 2004;38(4):529536. DOI http://dx.doi.org/10.1590/S0034$\underline{89102004000400008}$

Silva JLT, Barbosa DS, Oliveira JA, Guedes DP. Distribuição centrípeta da gordura corporal, sobrepeso e aptidão cardiorrespiratória: associação com sensibilidade insulínica e alterações metabólicas. Arq Bras Endocrinol Metabol. 2006;50(6):1034-40. DOI http://dx.doi.org/10. 1590/S0004$\underline{27302006000600009}$

Sposito A, Carmelli B, Fonseca FAH, Bertolami MC, Afiune NA, Souza AD. Sociedade Brasileira de Cardiologia. IV Diretrizes brasileiras sobre dislipidemias e prevenção da aterosclerose: Departamento de Aterosclerose da Sociedade Brasileira de Cardiologia. Arq Bras Cardiol. 2007;88(Supl.1):1-19.

VI Diretrizes Brasileiras de Hipertensão. Arq Bras Cardiol. 2010;95(1):I-III. DOI http://dx.doi.org/10.1590/S0066-

782X2010001700001 